\title{
Experimental measurements on transverse vibration characteristics of piezoceramic rectangular plates by optical methods
}

\author{
Chien-Ching $\mathrm{Ma}^{\mathrm{a}, *}$, Hsien-Yang Lin ${ }^{\mathrm{b}}$ \\ ${ }^{a}$ Department of Mechanical Engineering, National Taiwan University, Taipei, Taiwan 106, Republic of China \\ ${ }^{\mathrm{b}}$ Department of Mechanical Engineering, De Lin Institute of Technology, Tu-Cheng, Taiwan 236, Republic of China
}

Received 25 March 2004; received in revised form 21 September 2004; accepted 13 October 2004

Available online 28 December 2004

\begin{abstract}
This study provides two non-contact optical techniques to investigate the transverse vibration characteristics of piezoceramic rectangular plates in resonance. These methods, including the amplitudefluctuation electronic speckle pattern interferometry (AF-ESPI) and laser Doppler vibrometer (LDV), are full-field measurement for AF-ESPI and point-wise displacement measurement for LDV, respectively. The edges of these piezoceramic rectangular plates may either be fixed or free. Both resonant frequencies and mode shapes of vibrating piezoceramic plates can be obtained simultaneously by AF-ESPI. Excellent quality of the interferometric fringe patterns for the mode shapes is obtained. In the LDV system, a built-in dynamic signal analyzer (DSA) composed of DSA software and a plug-in waveform generator board can provide the piezoceramic plates with the swept-sine excitation signal, whose gain at corresponding frequencies is analyzed by the DSA software. The peaks appeared in the frequency response curve are resonant frequencies. In addition to these optical methods, the numerical computation based on the finite element analysis is used to verify the experimental results. Good agreements of the mode shapes and resonant frequencies are obtained for experimental and numerical results.
\end{abstract}

(C) 2004 Elsevier Ltd. All rights reserved.

\footnotetext{
${ }^{*}$ Corresponding author. Tel.: + 886223659996 : fax: +886223631755.

E-mail addresses: ccma@ntu.edu.tw (C.-C. Ma), sylin@sitc.edu.tw (H.-Y. Lin).
} 


\section{Introduction}

Plates are structural elements of great importance and are used extensively in all fields of engineering applications such as aerospace and electronic industry. There have been extensive studies on the vibration of classical plates for various shapes, boundaries, and loading conditions for nearly two centuries. The analysis methods for vibrations of plates can be classified into three types, which are analytical [1], numerical [2,3] and experimental [4]. The analytical method is restricted to problems with one pair of opposite simply supported edges. For the plates with other boundary conditions, the exact solutions generally cannot be obtained by this method. As regards the numerical method, it can solve very complicated plate problems, but a large number of data needed to be processed and the enlarged computing time for increased accuracy are the serious drawbacks. The experimental methods commonly used for vibration analysis are the modal analysis and the spectrum analyzer; they are point-wise measurement techniques and generally are used in conjunction with certain types of accelerators and shakers. These point-wise techniques have suffered from irregular mode shape estimation as a result of lengthy data acquisition period. Hence, they are very time-consuming for vibration mode shapes extraction. Therefore, the need for experimental techniques with the characteristics of much faster and full-field configuration is the first motivation for the study presented herein.

Since Curie brothers discovered the piezoelectric effect in 1880, piezoelectric materials have been extensively used as major elements in electromechanical transducers, resonators, sensors, actuators, and ultrasonics, etc. In the past few years, the discovery of polarized ceramics especially of lead zirconate titanate (PZT) evolved the development of piezoelectric applications. Piezoceramic materials, because of their low cost, better piezoelectric activity and the ease with which the desired shape is machined, have gradually replaced conventional piezoelectric crystals. The application of piezoceramic materials as transducers for energy transformation or resonators for ultrasonics especially in the form of plates or disks is carried out in many engineering fields. A prominent feature of utilizing piezoceramic plates or disks is the study of the full-field and realtime vibration behavior. There have been a number of technical papers proposed to find the vibration characteristic of piezoelectric plates. Holland [5] used the Rayleigh-Ritz method to study the extensional modes of rectangular piezoelectric plates and classified them into four distinct symmetry types. Yang and Batra [6] proposed a systematic method that is based on an abstract formulation of the three-dimensional theory of piezoelectricity to analyze the eigenvalue problem associated with free vibrations of a finite piezoelectric body. Rogacheva [7] presented a comprehensive treatment for problems arising in the construction of applied theory of piezoelectric shells and plates. Chen et al. [8] presented a non-dimensional-state equation for the transversely isotropic piezoelectric body and the free vibration of a simply supported rectangular plate is analyzed. Most of the works in the literature are either theoretical or numerical analysis. Few results use experimental technique, especially the non-contact optical techniques, to investigate the vibration characteristics of piezoceramic plates. The lack of the fullfield experimental investigation and numerical analysis of vibration modes for piezoceramic plates is the second motivation for the study presented herein. Therefore, in this study, the first optical technique called amplitude-fluctuation electronic speckle pattern interferometry (AF-ESPI), which has the advantages of non-contact, real-time observation, and high precision, is adopted to 
investigate the vibration characteristics of piezoceramic rectangular plates with completely free and completely fixed boundaries.

ESPI is also known as TV-holography which combines the techniques of image plane holography and speckle interferometry. The recorded work on ESPI was first proposed by Butters and Leendertz [9] who investigated the out-of-plane displacement of a vibrating disk. It has become a well-established technique for vibration analysis and is applied to many engineering problems. Løkberg and Malmo [10] demonstrated that the ESPI could be used to measure vibration of objects placed at long distances from the ESPI head. Chai et al. [11] combined TV-holography with FEM to study the vibration characteristics of laminated composite plates. Wang et al. [12] proposed the AF-ESPI for vibration measurement to increase the visibility of time-averaging interferometric fringe patterns. Ma and his coworker $[13,14]$ further used the AF-ESPI and the impedance analysis to investigate three-dimensional volume vibrations of piezoelectric materials. In their works, the interferometric fringe patterns obtained by AF-ESPI are clearly displayed with high quality for identification of resonant frequencies and mode shapes. The out-of-plane and in-plane vibration modes are coupled for three-dimensional vibrations. However, for the two-dimensional vibrations of plates, the out-of-plane and in-plane vibration modes are uncoupled. Huang and Ma [15] investigated the out-of-plane (three modes) and in-plane (eight modes) vibration characteristics for rectangular piezoceramic plates with completely free boundary and indicated that the out-of-plane vibration modes with low resonant frequencies cannot be measured by the impedance analysis. However, excellent agreement of the resonant frequencies (from 35000 to $139100 \mathrm{~Hz}$ ) for the in-plane modes measured by AF-ESPI and impedance analysis is found by Huang and Ma [15]. Hence, an alternative experimental measuring technique for determination of resonant frequencies for out-of-plane vibration modes of piezoceramic plates is essential for comparison with the results obtained by AF-ESPI.

The second optical technique employed in this work is the recently developed laser Doppler vibrometer (LDV) incorporating with a dynamic signal analysis (DSA) hardware and software [16]. Generally, the LDV measures the moving velocity or displacement of an object by detecting the frequency shift of the laser. It has been the most favorite instrument for precision dynamic parameters measurement due to its non-contact nature, high accuracy, high spatial density and ultra-high resolution better than nanometer. This method has been used to meet various metrology needs for many years $[17,18]$. However, most of the LDV systems commercially available have suffered from cumbersome and time-consuming analysis work because of the lengthy data processing for dynamic study. Hence, in the study, the dynamic signal analysis module incorporated in the LDV system enables the excitation signal to be emitted and the dynamic signal measured by LDV to be processed easier and faster. It appears that such a measurement system enables the dynamic study of out-of-plane vibrations for piezoceramic plates with various boundaries.

By using these two optical measurement techniques, the resonant frequencies and mode shapes of vibrating piezoceramic plates with completely free and completely fixed boundary conditions are achieved thoroughly. In addition, in light of the results presented in this work, numerical computations based on the finite element method (FEM) utilizing ABAQUS commercial software package [19] are also made. The excellent agreement of the experimental data with numerical results reveals that the presented methodologies in measuring vibration response of piezoceramic plates own an available exactitude. 


\section{Principles of experimental techniques}

\subsection{AF-ESPI}

The time-averaging method is the most familiar technique of ESPI used for vibration analysis. The name of "time-averaging" indicates that the vibration measurement includes many periods of object motions during the camera frame period. The light intensity of the image is usually detected by a charge-coupled device (CCD) camera. Two different optical setups are commonly used (outof-plane and in-plane sensitivity) for the vibration measurement. Fundamentals with regards to the AF-ESPI method for in-plane and out-of-plane measurements can be found in Ref. [13,14]. Since the piezoceramic plate used in this work is thin, it will display large transverse (out-of-plane) motion compared to any possible in-plane components, especially in the low resonant frequencies. Hence, only the out-of-plane sensitivity optical setup is used in this study. As shown in Fig. 1, a He-Ne Laser (Uniphase 1135P) with $30 \mathrm{~mW}$ and wavelength $\lambda=632.8 \mathrm{~nm}$ is used as the coherent light source. The emitting laser beam is split into two parts by a variable beamsplitter (BS). One beam is directed toward the tested piezoceramic plate and then reflects to the CCD camera acting as the object beam. The second one served as a reference beam is illuminated on the surface of a reference plate and reflects into the CCD camera via the beamsplitter (BS). The object and reference beams are combined into the CCD sensor array through a zoom lens (Nikon MicroNikkor $55 \mathrm{~mm}$ ). It is important to note that the optical path and the light intensity of these two beams should remain identical in the experimental setup. A CCD camera (Pulnix TM-7CN) and a

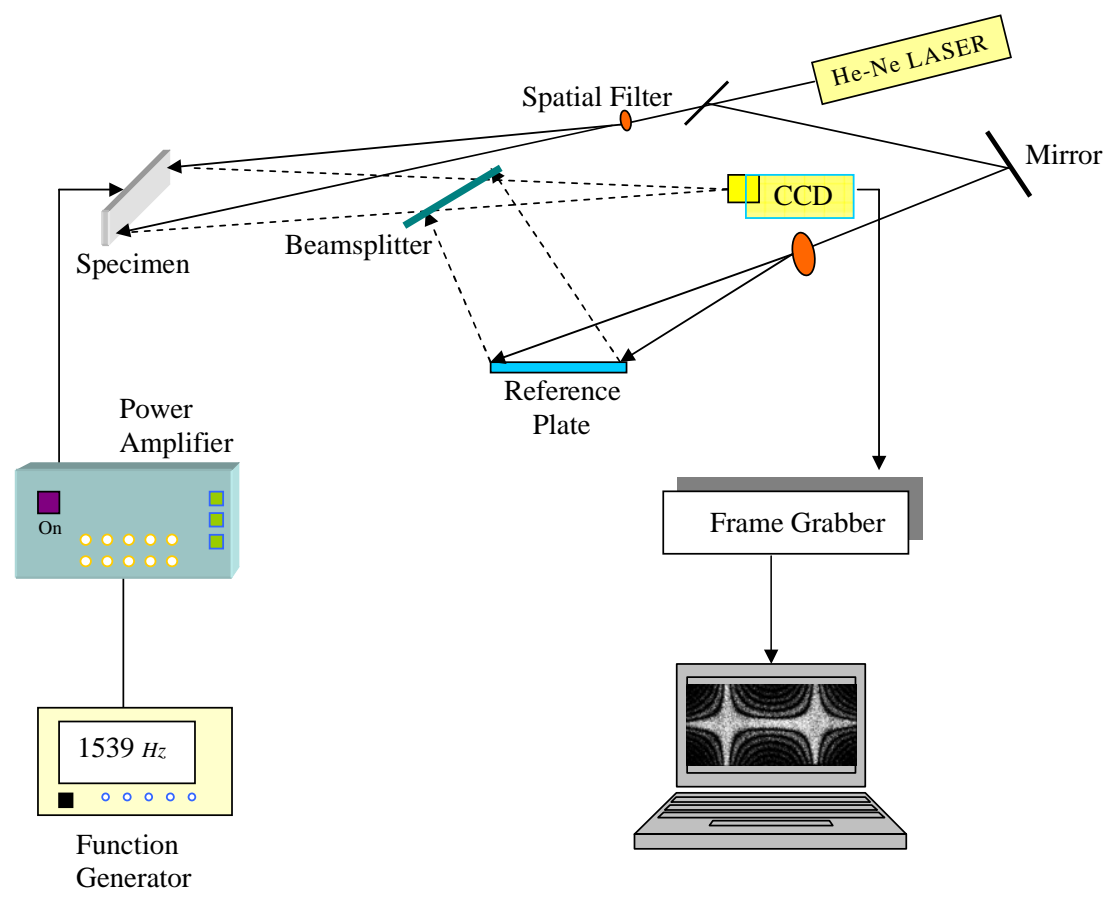

Fig. 1. Optical setup of ESPI for the out-of-plane measurement. 
frame grabber (Dipix P360F) with a digital signal processor on board are used to record and process the images obtaining from interferogram of the object and reference beams. Once the object vibrates, the interferogram recorded by the CCD camera is stored in an image buffer as a reference image. Then the next frame is grabbed and is subtracted by the image processing system. The CCD camera converts the intensity distribution of the interference pattern of the object into a corresponding video signal at 30 frames per second. The signal is electronically processed and finally converted into an image on the video monitor. The interpretation of the fringe image is similar to the reading of a displacement contour. To achieve a sinusoidal output, a function generator (Hewlett Packard, HP-33120A) connected to a power amplifier (NF Electronic Instruments 4005 type) is employed as an input source, which generates periodical exciting force to the specimen.

The detailed experimental procedure of the AF-ESPI technique is performed as follows. First, a reference image is taken after the specimen vibrates, then the second image is taken, and the reference image is subtracted by the image processing system. If the vibrating frequency is not near the resonant frequency, only randomly distributed speckles are displayed and no fringe patterns will be shown. However, if the vibrating frequency is in the neighborhood of the resonant frequency, stationary distinct fringe patterns corresponding to this vibration mode will be observed. Then the function generator is cautiously and gradually turned, the number of fringes will increase and the fringe pattern will become clearer, but the position of nodal lines will not change as the resonant frequency is approached. From the aforementioned experimental procedure, the resonant frequencies and the corresponding mode shapes can be determined at the same time by using the AF-ESPI optical system.

When the two images are subtracted by the image processing system, the resulting image intensity can be expressed as

$$
I \propto\left|J_{0}(\Gamma A)\right|,
$$

where

$$
\Gamma=\frac{2 \pi}{\lambda} A(1+\cos \theta),
$$

in which $J_{0}$ is a zero-order Bessel function of the first kind, $\theta$ is the angle between object light and observation direction, $A$ is the vibration amplitude. The basic theory and detailed derivation of AF-ESPI method can be found in Ref. [13-15].

\section{2. $L D V$}

The second optical technique is a LDV. To measure the vibration characteristic of a rectangular piezoceramic plate, a DSA software and a waveform generator board are integrated into the LDV system to become the LDV-DSA system. Namely, the LDV-DSA system combines the measuring capacity of LDV with the analysis capacity of DSA. In this system, the waveform generator board is plugged in the personal computer to send a series of sine wave with varied frequencies and voltages to excite the piezoceramic plate, where the sweeping range of frequencies and corresponding excitation voltage for certain frequency range can be set by users. At the same time, the LDV measures the dynamic response of the piezoceramic plates. For DSA calculation, 
the swept-sine excitation signal is taken as input and the response measured by LDV is taken as output; after the fast Fourier transform (FFT) processing of the input and output with the DSA software, the ratio of output/input ("gain") and the phase relation between the output response and input signal are obtained. After the so-called the DSA calculations, the result chart that shows the frequency response curve can be obtained directly. The test block diagram is shown in Fig. 2. A description of LDV components and operating principle is given in complete detail by Lee and $\mathrm{Wu}$ [16] and is briefly outlined below. The LDV-DSA system utilizes circular polarization interferometry instead of acoustic optical modulator (AOM) that is generally adopted in the conventional LDV system. The circular polarization interferometry can significantly reduce the size of the vibrometer and avoid the RF electromagnetic interference effects associated with the AOM. The so-called "circular polarization interferometry" technique is that the two returned interference beams are converted to right and left circularly polarized light by the use of a quarter wave plate and digital signal processing with built-in phase decoding algorithms, thus the system can be designed to measure dynamic displacement and vibration responses of almost any objects. The optical system, depicted in Fig. 3, is based on the principle of the Michelson interferometer and the Doppler effect to measure the dynamic displacement. In this figure, a stabilized He-Ne laser is split into two interfered arms by the polarization BS (PBS1) within the optical head. Beam 1 passes through BS (PBS2) and is focused to a point on the tested object. For absolute displacement measurement, a corner cube reflects beam 2 acting as a reference beam. As beam 1 and beam 2 are split by the PBS1, the two beams will possess "p" and "s" polarization states, respectively. These two returning beams after passing through PBS1 are linearly polarized and are orthogonal with each other, and then are reflected by a BS. A quarter waveplate oriented at $45^{\circ}$ denoting QWP(45) is used to transform the two returning light beams into a right circularly polarized light and a left circularly polarized light. The sum vector of these

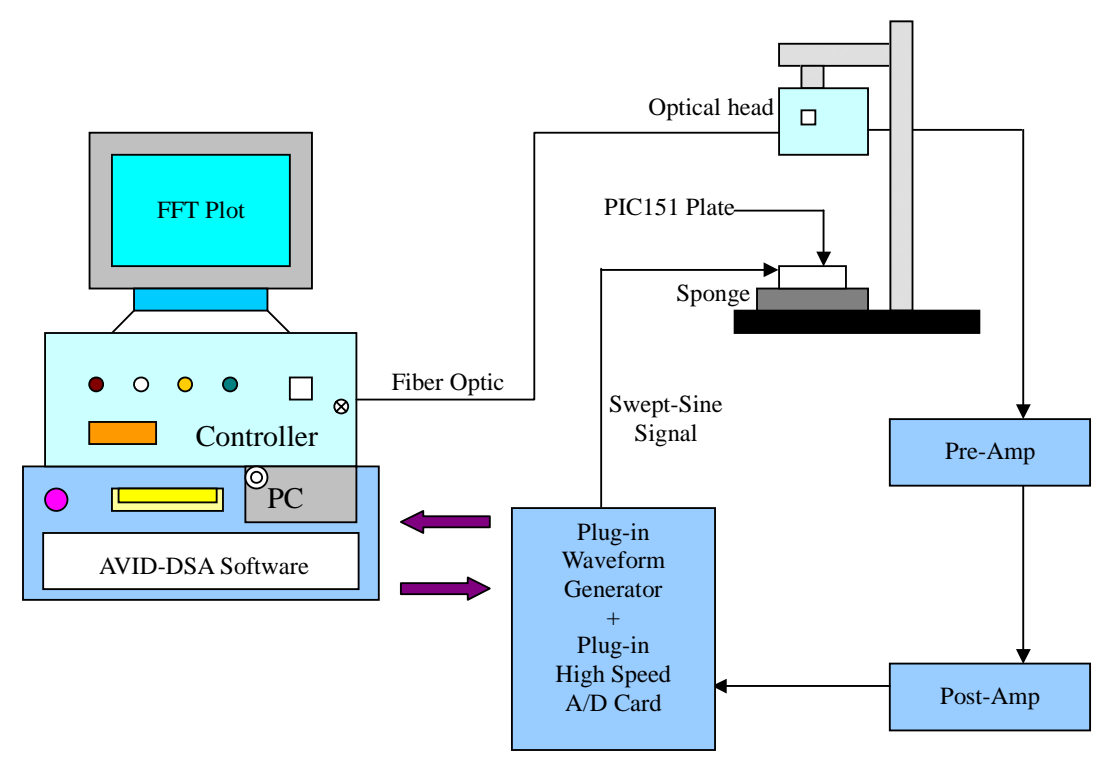

Fig. 2. Schematic diagram of the LDV-DSA measurement system (Ref. [16]). 


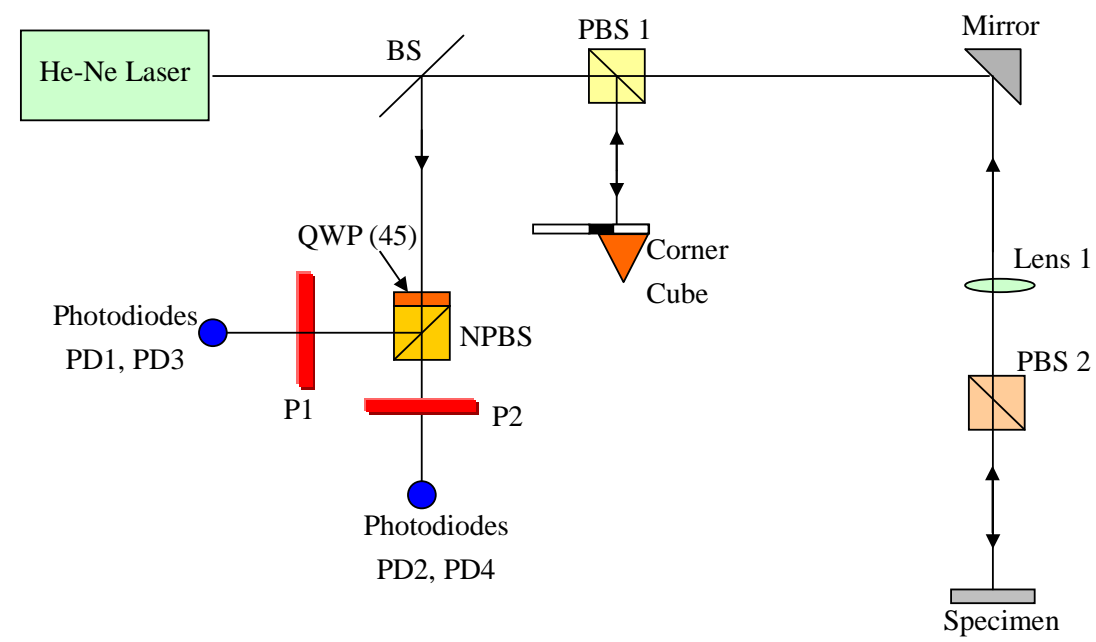

Fig. 3. Schematic diagram of the LDV optical setup for absolute displacement measurement (Ref. [16]).

two circular polarized light beams can be viewed as a linearly polarized light with its polarization axis locating at a specific angle. Because the movement or displacement of the tested object will result in different propagation speed of the left- and right-hand polarized light, the two combined beams remain behaving like a linearly polarized light but with an inclined angle relative to a horizontal axis. Then the resultant linearly polarized light beam is divided into two interfered light beams by a non-polarization BS (NPBS). Two polarizers P1 and P2, situated at $45^{\circ}$ apart, transform the polarization states into quadrature intensity signals, which can be detected by photodiodes. In order to eliminate the average DC level from the quadrature signals and to increase the quality of the signals, a pair of PBSs and four photodiodes PD1-PD4 are designed to produce four signals. The four quadrature signals detected by photodiodes can be expressed as [16]

$$
\begin{array}{ll}
I_{1} \propto\left[1+\sin \left(2 \pi f_{d} t+\theta_{0}\right)\right], & I_{2} \propto\left[1+\cos \left(2 \pi f_{d} t+\theta_{0}\right)\right], \\
I_{3} \propto\left[1-\sin \left(2 \pi f_{d} t+\theta_{0}\right)\right], & I_{4} \propto\left[1-\cos \left(2 \pi f_{d} t+\theta_{0}\right)\right],
\end{array}
$$

where $f_{d}$ is the Doppler phase shift due to the object movement, and $\theta_{0}$ represents the relative phase difference between beam 1 and beam 2 .

The signals $P$ and $Q$ standing for $\left(I_{1}-I_{3}\right)$ and $\left(I_{2}-I_{4}\right)$, respectively, are amplified and shaped by the circuit board. These two signals differing by $90^{\circ}$ phase angle are put into an analog-to-digital converter. By plotting these two signals $P$ and $Q$ on a $X-Y$ plane, a Lissajous graph is constructed. That is, a circular pattern is formed when the phase difference between the two beams changes by $360^{\circ}$ (or the tested object displaces half wavelength). Decoding the angle for any specific point on this circle is known as the $P / Q$ signal decoding. The signal decoding algorithm involves many matrix operations; the detailed derivation can be found in Ref. [16]. The displacement $D$ can be accurately determined by the formula

$$
D=\frac{\theta}{2 \pi} \times \frac{\lambda}{2} .
$$


During the period of frequency sweeping, the excitation signal with varied frequency is sent to the piezoceramic plate; meanwhile, the LDV measures the response of the piezoceramic plate. After the FFT processing of the input and output, the "gain" for a specific point on the upper surface of the piezoceramic plate at this frequency span is calculated; hence, the frequency response curve can be obtained rapidly and easily. The resonant frequencies of the piezoceramic plates are thus easily identified from the corresponding peaks of the frequency response curve.

\section{Experimental and numerical results}

The vibration characteristics of two rectangular piezoceramic plates with completely free and completely fixed boundaries are investigated. The plates have the size of $50 \times 20 \times 0.26 \mathrm{~mm}$ and are made of $\mathrm{Pb}(\mathrm{Zr} \mathrm{Ti}) \mathrm{O}_{3}$ ceramics, and the model number is PIC-151 (Physik Instrumente). The material properties of the piezoceramic plates are listed in Table 1. The polarization axis is in the thickness direction, and two opposite faces of the piezoceramic plates are completely coated with silver electrodes. A self-arranged time-averaging ESPI system as shown in Fig. 1 is used to perform the experimental measurements for resonant frequencies and corresponding mode shapes. Figs. 4 and 5 show the first ten mode shapes of the PIC-151 piezoceramic plates obtained by AF-ESPI and FEM for completely free and completely fixed boundaries, respectively. To simulate the completely free boundary and completely fixed boundary experimentally, the piezoceramic plate is put on a very soft sponge for the free boundary approximation and is glued and clamped on four sides for the fixed boundary approximation. Numerical computations of resonant frequencies as well as mode shapes are implemented by the commercially available software ABAQUS finite element package [19]. A three-dimensional solid piezoelectric element "C3D20RE", which is a 20-node quadratic brick with reduced integration, is adopted to analyze the vibration of piezoceramic plates. The electrical potentials on the top and bottom surfaces of the piezoceramic plates are set to "zero" for simulation of closed-circuit case. For the finite

Table 1

Material properties of the PIC-151 piezoelectric plate

\begin{tabular}{ll}
\hline Quality & PIC-151 \\
\hline$C_{11}^{E}\left(\mathrm{~N} / \mathrm{m}^{2}\right)$ & $10.76 \times 10^{10}$ \\
$C_{12}^{E}$ & $6.313 \times 10^{10}$ \\
$C_{13}^{E}$ & $6.386 \times 10^{10}$ \\
$C_{33}^{E}$ & $10.04 \times 10^{10}$ \\
$C_{44}^{E}$ & $1.962 \times 10^{10}$ \\
$C_{66}^{E}$ & $2.224 \times 10^{10}$ \\
$e_{31}(\mathrm{~N} / \mathrm{Vm})$ & -9.52 \\
$e_{33}$ & 15.14 \\
$e_{15}$ & 11.97 \\
$\rho\left(\mathrm{kg} / \mathrm{m}^{3}\right)$ & 7800 \\
$\varepsilon_{11}^{E} / \varepsilon_{0}$ & 1111 \\
$\varepsilon_{\varepsilon 11}^{E} / \varepsilon_{0}$ & 925 \\
$\varepsilon_{0}(\mathrm{~F} / \mathrm{m})$ & $8.85 \times 10^{-12}$ \\
\hline
\end{tabular}




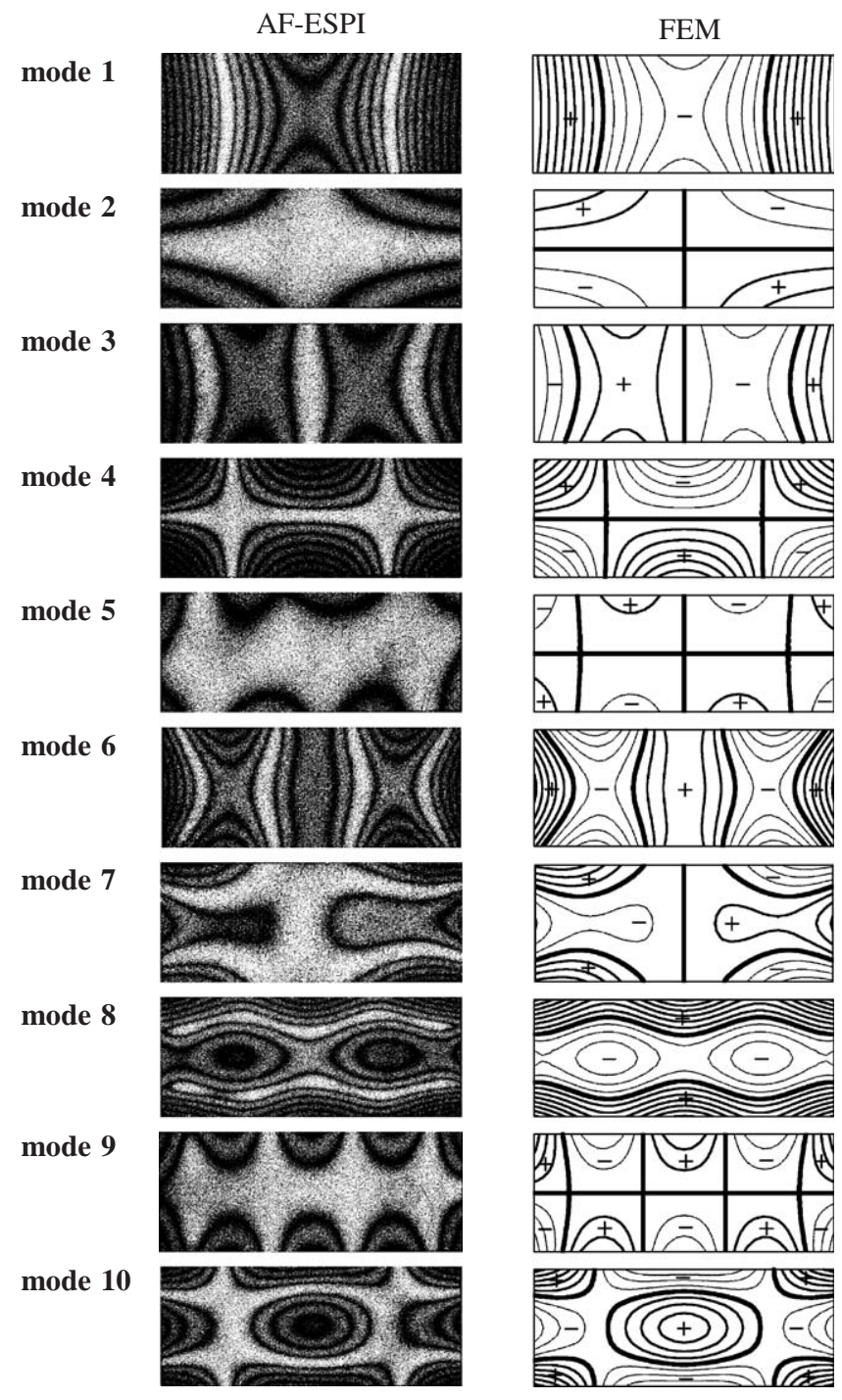

Fig. 4. Mode shapes of the piezoceramic plate with completely free boundary.

element results as shown in the right-hand side of Figs. 4 and 5, the dashed lines and the "-" sign denote the concave displacements, while the solid lines and the "+" sign denote convex displacements. The transition from the solid lines to dashed lines corresponds to a zero displacement line or a nodal line that is represented as a bold line. The zero-order fringes, which are the brightest fringes on the experimental results, represent the nodal lines of the piezoceramic plates at resonant frequencies. The rest of the dark fringes are contours of constant displacement, which can be quantitatively calculated by $\mathrm{J}_{0}(\Gamma A)=0$ as indicated in equation (1). We use $\theta=10^{0}$ for the experimental setup and $\lambda=632.8 \mathrm{~nm}$; the related vibration amplitudes for the first five dark fringes are $0.12,0.28,0.44,0.6$ and $0.76 \mu \mathrm{m}$. Hence, the sensitivity of the vibration 


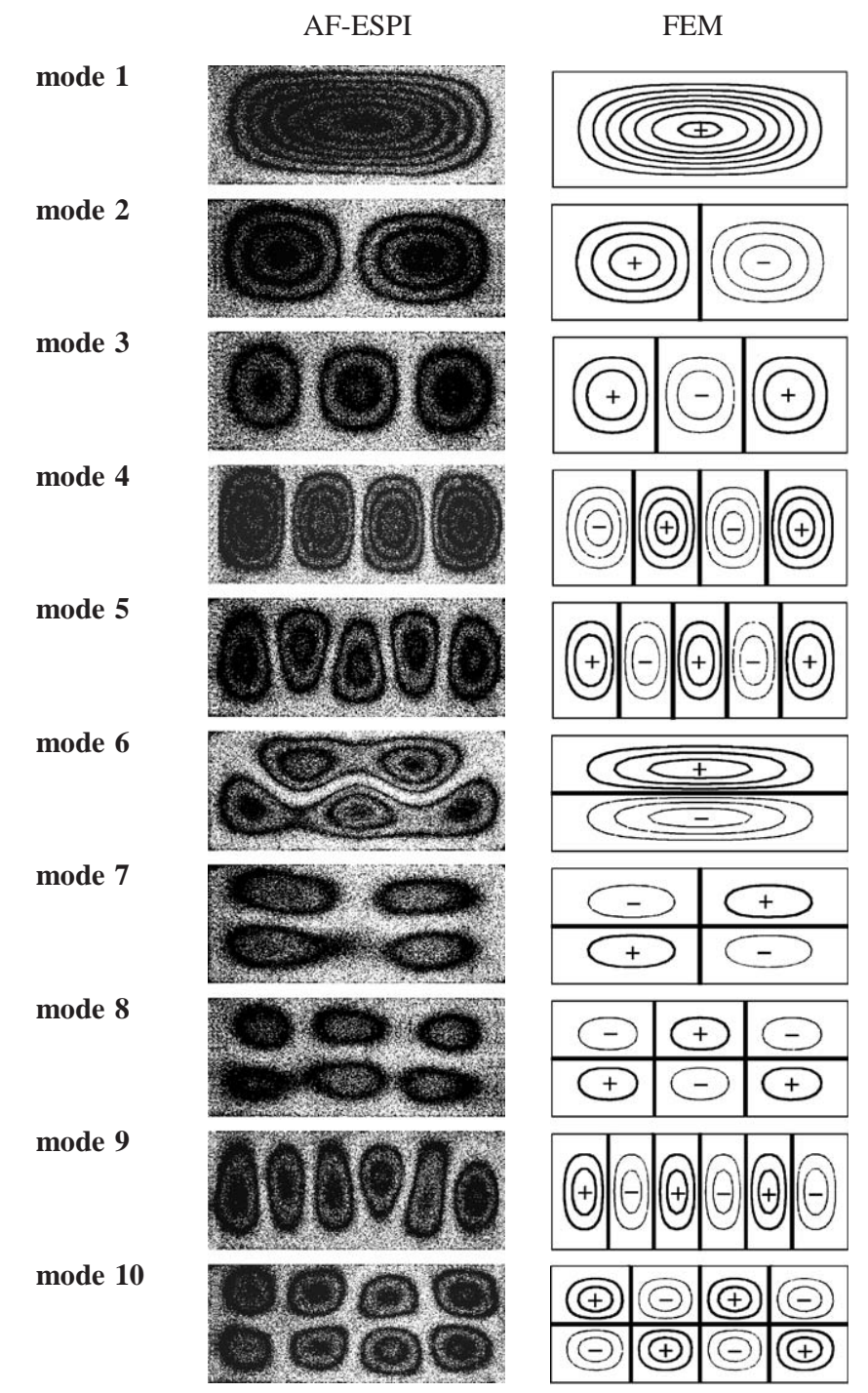

Fig. 5. Mode shapes of the piezoceramic plate with completely fixed boundary.

displacements obtained in this study is submicrometer. The mode shapes obtained by experimental results can be checked by the nodal lines and fringe patterns with the numerical finite element calculations, and excellent agreements are found.

The frequency responses of the completely free and completely fixed boundaries obtained by LDV-DSA system are shown in Figs. 6 and 7, respectively. The sweeping frequencies are set from 10 to $3110 \mathrm{~Hz}$ for completely free case, and from 10 to $8510 \mathrm{~Hz}$ for completely fixed case. The excitation signal is transmitted to the piezoceramic plate by two thin wires that are connected on the electrode surfaces. The two plots shown in Figs. 6 and 7 take the form of "frequency-gain" with Hanning window for reducing data leakage and increasing measurement accuracy; the 


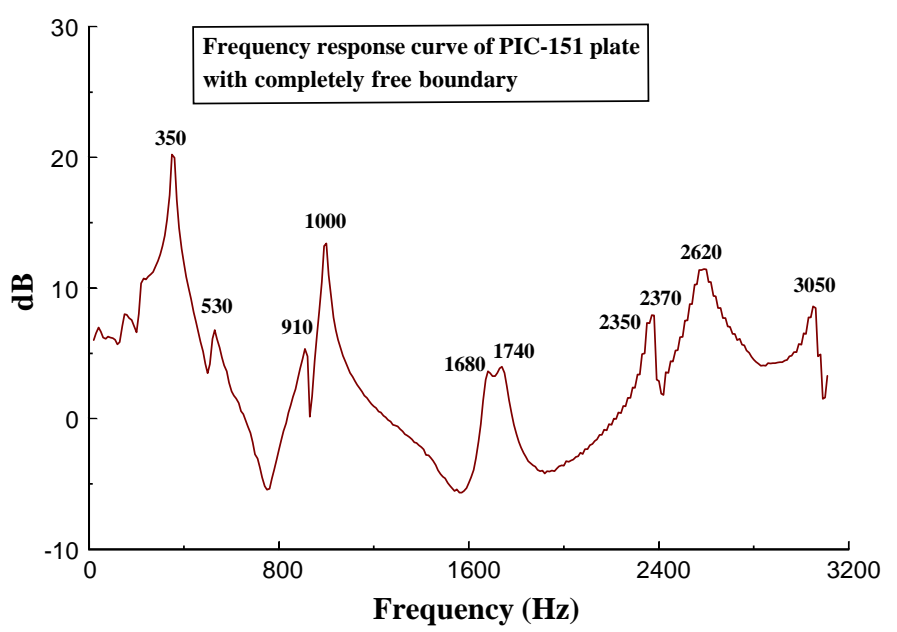

Fig. 6. Frequency response curve of the piezoceramic plate with completely free boundary.

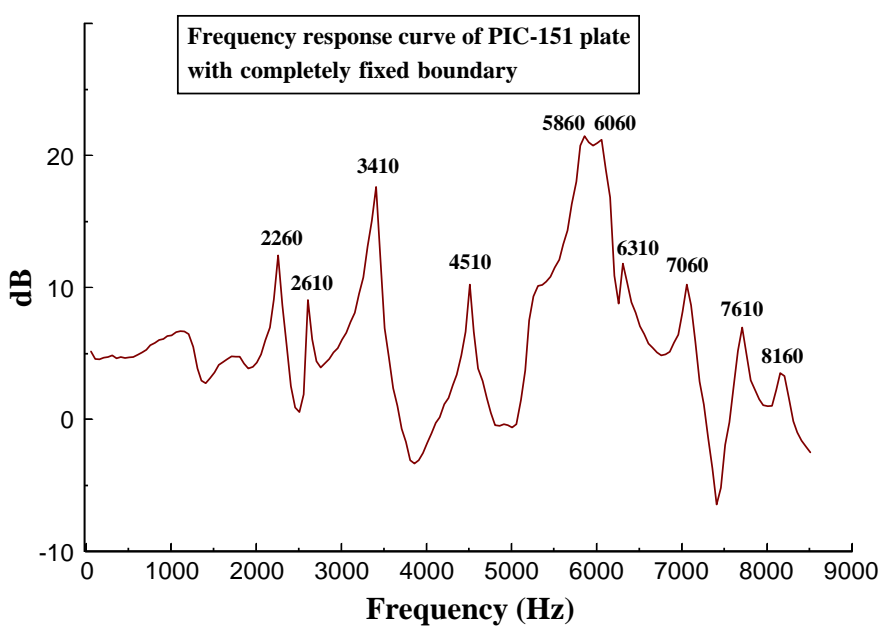

Fig. 7. Frequency response curve of the piezoceramic plate with completely fixed boundary.

vertical axes of these two plots are in logarithmic scale. The first ten peaks shown in Figs. 6 and 7 corresponding to the first ten resonant frequencies are in excellent agreement with the measured values by AF-ESPI. Tables 2 and 3 tabulate the resonant frequencies obtained from experimental and FEM methods. The measured results of resonant frequencies by two optical methods agree well with numerical predictions. The discrepancy of resonant frequencies between AF-ESPI and LDV-DSA is about $1 \%$ and is much smaller than that between AF-ESPI and the FEM. This discrepancy is probably due to the material properties measurement and the boundary condition of the specimen. Compared the experimental values with the FEM results, the largest error for completely free boundary occurs at the first and the second modes. It is believed that the effect of soft sponge has a distinct influence on lower resonant frequencies. However, all of the 
Table 2

Resonant frequencies obtained from experimental measurement and FEM calculations for completely free boundary (error is compared to AF-ESPI values)

\begin{tabular}{llll}
\hline Method & $\begin{array}{l}\text { AF-ESPI }(\mathrm{Hz}) \\
\text { Resonant frequency }\end{array}$ & $\begin{array}{l}\text { LDV }(\mathrm{Hz}) \\
\text { Resonant frequency (Error \%) }\end{array}$ & $\begin{array}{l}\text { FEM }(\mathrm{Hz}) \\
\text { Resonant frequency (Error \%) }\end{array}$ \\
\hline Mode 1 & 362 & $350(-3.315)$ & $324.4(-10.387)$ \\
Mode 2 & 528 & $530(0.379)$ & $451.6(-14.470)$ \\
Mode 3 & 919 & $910(-0.979)$ & $916.6(-0.261)$ \\
Mode 4 & 1002 & $1000(-0.200)$ & $989(-1.297)$ \\
Mode 5 & 1683 & $1680(-0.178)$ & $1695(0.713)$ \\
Mode 6 & 1757 & $1740(-0.968)$ & $1780.3(1.326)$ \\
Mode 7 & 2320 & $2350(1.293)$ & $2386.3(2.856)$ \\
Mode 8 & 2373 & $2370(-0.126)$ & $2403.6(1.290)$ \\
Mode 9 & 2612 & $2620(0.306)$ & $2639.6(1.057)$ \\
Mode 10 & 3063 & $3050(-0.424)$ & $3071.9(0.291)$ \\
\hline
\end{tabular}

Table 3

Resonant frequencies obtained from experimental measurement and FEM calculations for completely fixed boundary (error is compared to AF-ESPI values)

\begin{tabular}{llll}
\hline Method & $\begin{array}{l}\text { AF-ESPI }(\mathrm{Hz}) \\
\text { Resonant frequency }\end{array}$ & $\begin{array}{l}\text { LDV }(\mathrm{Hz}) \\
\text { Resonant frequency (Error \%) }\end{array}$ & $\begin{array}{l}\text { FEM (Hz) } \\
\text { Resonant frequency (Error \%) }\end{array}$ \\
\hline Mode 1 & 2232 & $2260(1.254)$ & $2551.9(14.332)$ \\
Mode 2 & 2589 & $2610(0.811)$ & $3001.6(15.937)$ \\
Mode 3 & 3342 & $3410(2.034)$ & $3824.5(14.437)$ \\
Mode 4 & 4455 & $4510(1.235)$ & $5043(13.199)$ \\
Mode 5 & 5891 & $5860(-0.526)$ & $6650.8(12.898)$ \\
Mode 6 & 6078 & $6060(-0.296)$ & $6810.3(12.048)$ \\
Mode 7 & 6240 & $6310(1.122)$ & $7277.1(16.620)$ \\
Mode 8 & 7058 & $7060(0.028)$ & $8081.1(14.496)$ \\
Mode 9 & 7688 & $7610(-1.015)$ & $8637.5(12.350)$ \\
Mode 10 & 8148 & $8160(0.147)$ & $9246.6(13.483)$ \\
\hline
\end{tabular}

experimental measured frequencies are lower than the numerical results for the completely fixed boundary. The main reason is that the fixed condition of the piezoceramic plate for the experimental setup is not ideally rigid.

A piezoelectric element that couples the mechanical and electric fields is required for FEM simulation. It is very cumbersome to do such analysis because too many material constants are involved in the simulation. In this study, we also investigate the influence of piezoelectric effect on the vibration behavior of plates. Three elements in ABAQUS software package, namely S8R5 shell element, C3D20R solid element and C3D20RE piezoelectric element, are used to model the vibration behavior of piezoceramic plates. In general, the analysis that uses the shell and solid elements is easier and much faster than that uses the piezoelectric element. Fig. 8 shows the resonant frequencies obtained by using different elements and it may underestimate the resonant frequencies by using the shell and solid elements. Only tiny difference is observed for lower 


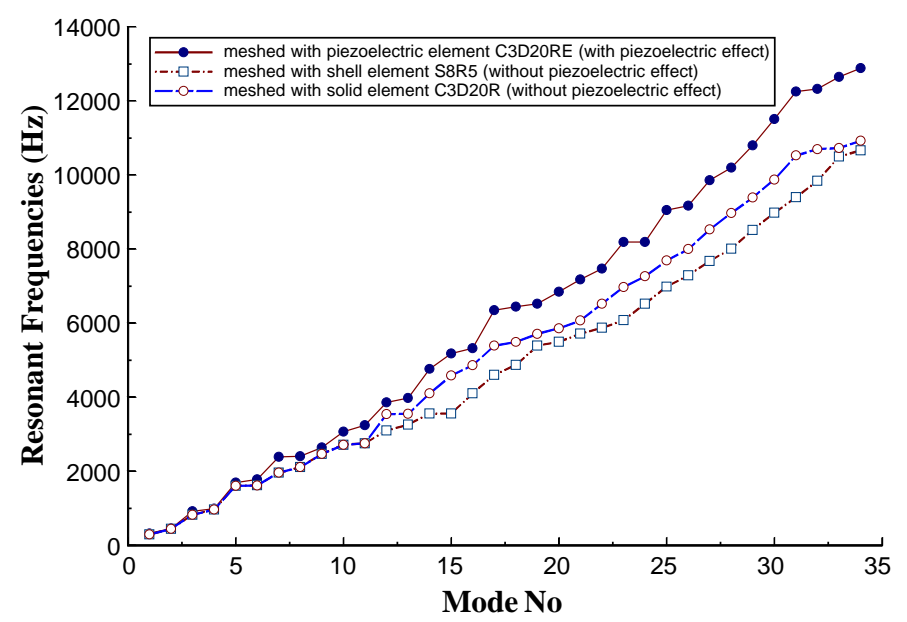

Fig. 8. Comparison of resonant frequencies obtained by FEM using the piezoelectric, shell and solid elements.

frequencies; but larger error may be introduced for higher frequencies using shell and solid elements.

\section{Conclusions}

It is known that the vibration characteristics of piezoceramic plates are important in many engineering applications. However, there are only few experimental results available for vibrating piezoceramic plates. This study has examined the resonant frequencies and mode shapes of vibrating piezoceramic plates by using AF-ESPI and LDV-DSA. It is shown that these two optical methods have the advantages of non-contact, real-time and high-resolution measurement for the vibration behavior of piezoceramic materials. Moreover, no equipment, transducer or sensor needs to be attached to the piezoceramic plate in order to record the vibration. According to our results, the resonant frequencies measured by AF-ESPI correlate well with LDV-DSA. AF-ESPI enables both the full-field mode shapes and resonant frequencies to be obtained at the same time, while the LDV-DSA system obtains the resonant frequencies rapidly and easily. The interferometric fringes of AF-ESPI are produced instantly by a video recording system. Two different types of boundary conditions are investigated and twenty mode shapes with excellent quality are demonstrated in this study. Numerical computations of resonant frequencies and mode shapes based on a finite element package are also performed, and excellent agreements for the modes shapes are obtained when compared with results obtained by AF-ESPI. However, resonant frequencies obtained by experimental methods for completely fixed boundary are typically lower than theoretical ones because one cannot get perfectly rigid clamped condition on experiment. These results also demonstrate that the optical techniques proposed herein are very applicable to many situations in engineering vibration analysis for piezoelectric materials with different boundary conditions. 


\section{Acknowledgements}

The authors gratefully acknowledge the financial support of this research by the National Science Council (Republic of China) under Grant NSC 89-2218-E-002-034.

\section{References}

[1] I.E. Harik, X. Liu, N. Balakrishnan, Analytic solution to free vibration of rectangular plates, Journal of Sound and Vibration 153 (1992) 51-62.

[2] W.Q. Chen, R.Q. Xu, H.J. Ding, On free vibration of a piezoelectric composite rectangular plate, Journal of Sound and Vibration 218 (1998) 741-748.

[3] T. Sakiyama, H. Matsuda, Free vibration of rectangular Mindlin plate with mixed boundary conditions, Journal of Sound and Vibration 113 (1987) 208-214.

[4] K.H. Low, G.B. Chai, T.M. Lim, S.C. Sue, Comparisons of experimental and theoretical frequencies for rectangular plates with various boundary conditions and added masses, International Journal of Mechanical Sciences 40 (1998) 1119-1131.

[5] R. Holland, Contour extensional resonant properties of rectangular piezoelectric plates, IEEE Transactions on Sonics and Ultrasonics 15 (1968) 97-105.

[6] J.S. Yang, R.C. Batra, Free vibrations of a piezoelectric body, Journal of Elasticity 34 (1994) 239-254.

[7] N.N. Rogacheva, The Theory of Piezoelectric Shells and Plates, CRC Press, Boca Raton, 1994.

[8] W.Q. Chen, R.Q. Xu, H.J. Ding, On free vibration of a piezoelectric composite rectangular plate, Journal of Sound and Vibration 218 (1998) 741-748.

[9] J.N. Butters, J.A. Leendertz, Speckle patterns and holographic techniques in engineering metrology, Optics and Laser Technology 3 (1971) 26-30.

[10] O.J. Løkberg, J.T. Malmo, Long-distance electronic speckle pattern interferometry, Optical Engineering 27 (1988) $150-156$.

[11] G.B. Chai, S.S. Chin, T.M. Lim, K.H. Hoon, Vibration analysis of laminated composite plates: TV-holography and finite element method, Composite Structures 23 (1993) 273-283.

[12] W.C. Wang, C.H. Hwang, S.Y. Lin, Vibration measurement by the time-averaging electronic speckle pattern interferometry method, Applied Optics 35 (1996) 4502-4509.

[13] C.H. Huang, C.C. Ma, Vibration characteristics for piezoelectric cylinders using amplitude-fluctuation electronic speckle pattern interferometry, AIAA Journal 36 (1998) 2262-2268.

[14] C.C. Ma, C.H. Huang, The investigation of three-dimensional vibration for piezoelectric rectangular parallelepipeds using the AF-ESPI method, IEEE Transactions on Ultrasonics, Ferroelectrics, and Frequency Control 48 (2001) 142-153.

[15] C.H. Huang, C.C. Ma, Experimental and numerical investigations of resonant vibration characteristics for piezoelectric plates, Journal of the Acoustical Society of America 109 (2001) 2780-2788.

[16] C.K. Lee, G.Y. Wu, High performance Doppler interferometer for advanced optical storage system, Japanese Journal of Applied Physics 38 (1999) 1730-1741.

[17] B.K.A. Ngoi, K. Venkatakrishnan, B. Tan, Laser scanning heterodyne interferometer for micro-components, Optics Communication 173 (2000) 291-301.

[18] O. Nishizawa, T. Satoh, X. Lei, Detection of shear wave in ultrasonic range by a laser Doppler vibrometer, Review of Scientific Instruments 69 (1998) 2572-2573.

[19] ABAQUS User's Manual, Version 5.5, Hibbit, Karlsson, and Sorensen, Inc., Pawtucket, RI, 1998. 